\title{
A hybrid agent based virtual organization for studying knowledge evolution in social systems
}

\author{
Sujatha Srinivasan ${ }^{1}$, Sivakumar Ramakrishnan ${ }^{2}$ \\ 1. Department of Computer Science, Cauvery College for Women, Trichy, Tamil Nadu, India. 2. Department of Computer \\ Science, AVVM Sri Pushpam College, Poondi, Tamil Nadu, India. \\ Correspondence: SujathaSrinivasan. Address: Department of Computer Science, Cauvery College for Women, Trichy, \\ Tamil Nadu, India, 620018. Telephone: 914-319-443-884-711. Email: ashoksuja03@yahoo.co.in.
}

Received: April 30, 2012

Accepted: September 20, 2012

Published: December 1, 2012

DOI : 10.5430/air.v1n2p99

URL: http://dx.doi.org/10.5430/air.v1n2p99

\begin{abstract}
Social modeling applies computational methods and techniques to the analysis of social processes and human behavior. Cultural algorithms (CA's) are evolutionary systems which utilize agent technology and which supports any evolutionary strategy like genetic algorithm, evolutionary algorithm or swarm intelligence or ant algorithms. CA's have been used for modeling the evolution of complex social systems, for re-engineering rule based systems, for data mining, and for solving optimization problems. In the current study a cultural algorithm framework is used to model an Agent Based Virtual Organization (ABVO) for studying the dynamics of a social system at micro as well as macro level. Research gap exists in defining a concrete and systematic method for evaluating and validating Agent Based Social Systems (ABSS). Also the knowledge evolution process at micro and macro levels of an organization needs further exploration. The proposed CA is applied to the problem of multi-objective optimization (MOO) of classification rules. The evolutionary knowledge produced by the agents in creating the rules is accepted into the belief space of the CA and macro evolution takes place. The belief space in turn influences the agents in successive generations. The rules created by the individuals and the knowledge sources created during evolution provide a concrete method to evaluate both the individuals as well as the whole social system. The feasibility of the system has been tested on bench mark data sets and the results are encouraging.
\end{abstract}

\section{Key words}

Cultural algorithm, Agent based social modeling, Multi-Objective optimization, Classification rule, Data mining, Virtual organization, Multi-agent system, Intelligent agents

\section{I ntroduction}

Wang et al. ${ }^{[1]}$ discuss the theoretical, methodological, and technological underpinnings of social computing. The authors have reviewed several major application areas, and have raised some key research issues. They identify four main areas of application namely the creation of Web 2.0 services and tools to support effective online communication for social communities, the second is entertainment software, which focuses on building intelligent entities that can interact with human users. The third application area discussed is the business and public sector, which includes various e-business, healthcare, economic, political, and digital government systems, as well as artificial engineering systems in domains of significant societal impact. A fourth application area is forecasting, which includes a variety of predictive systems for planning, evaluation, and training. The third and fourth areas of application form the background of our proposed work 
where a virtual organization is proposed for studying the knowledge evolution both at micro and macro levels. In particular a virtual organization containing agents with different cognitive traits and beliefs in a cultural algorithm framework applied in solving a multi criteria decision making problem forms the basis of the study.

Li et al. ${ }^{[2]}$ have reviewed literature on Agent Based Social Simulation and Modeling in Social Computing. The authors identify key future research directions that include conflict and cooperation between agents, trust and norm formation dynamics, modeling social and organizational structure, group decision making and collective behavior, emergence and evolution of organizations, among others with the design of human-like cognitive agents and virtual organizations being predicted by them as hot research direction in agent-based social simulation. However, they state that validation methodology is not yet mature.

$\mathrm{CaO}^{[3]}$ presents an overall picture of agent-mining integration and interaction as an emerging area. The author summarizes the main driving forces, complementary essence, disciplinary framework, applications, case studies, and trends and directions, with special emphasis on agent-driven data mining (DM), data mining-driven agents, and mutual issues in agent- mining. The author concludes that agent-mining is emerging as a new area in the scientific family and that both agent technology and data mining can greatly benefit from agent-mining, and that there is a promising outcome of agent-mining integration and interaction which will result in additional advancement in intelligent information processing and systems. Moreover the author states that DM techniques such as association rule extraction have no equivalent in agent systems which also form the basis of the proposed system.

A main drawback of ABSS's as found in the literature is their evaluation and validation. Research gap exists in defining a concrete, systematic and efficient method for evaluating the agents and the agent based systems ${ }^{[1-3]}$. The real world organizations are evaluated based on the products they produce or services they provide. The proposed ABVO is designed in such a way as to produce user specific classification rules. The agents in the system produce classification rules according to user specified rule metrics using their traits and beliefs thus driving the micro evolution, while the system chooses the best rules which are presented to the user and uses the gained knowledge to influences successive generations thus driving the macro evolution. The user can thus evaluate the organization as a whole at a macro level or the individual agents in the system at the micro level based on the products they produce namely the rules. Moreover the beliefs of the agents are defined by the type of knowledge they use. CA has a basic set of five different types of knowledge found in various animal species including human beings. Thus the proposed system integrates Social Computing with Data mining to create an Agent Based Virtual Organization (ABVO) for producing decision making rules. The proposed system thus provides a systematic and principled approach for evaluating agent based systems by applying the ABVO to solve a real world problem of rule mining.

$\mathrm{Cao}^{[3]}$ argues that research and development on agent-mining is very promising and worthy of substantial efforts by both established and new researchers. Hence Data mining with Social agents is a new area yet to be explored. Therefore an attempt has been made in the current study to combine the strengths of Intelligent Agents, Evolutionary Algorithms, Data mining and finally Human Computer Interaction to model an Agent Based Virtual Organization designed using a Cultural Algorithm framework. The agents in the proposed ABVO uses evolutionary Multi-Objective optimization techniques and user specified parameter values in creating a set of classification rules with differing tradeoffs to be presented to the user. The Multi-agent system is applied to solve the problem of classification rule mining as a multi-objective optimization problem. The agents in the system produce classification rules and agent's reproduction of rules using the genetic strategy of selection of chromosomes from the different knowledge sources using crossover and mutation form the micro evolution. The macro evolution is defined by the acceptance of best rules into the belief space. The performance of the $\mathrm{ABVO}$ is evaluated using the number of dominators returned, the metric values of these rules represented as the objective vector and in classifying unknown test data instances. Knowledge evolution thus takes place by producing new rules, accepting of best individuals into the belief space and the influence of the updated belief space in producing better rules. The accuracy of these rules in classifying unknown test data instances provide a systematic and principled approach for 
evaluating agent based systems. Section 2 defines the problem, the aims of the study and related work.The proposed ABVO for studying the dynamics of knowledge evolution is described in section 3. Section 4 discusses the experiments and results obtained by using the proposed ABVO on benchmark data set. Section 5 concludes with future work.

\section{Problem and related work}

\subsection{The problem}

Given a data source and multiple objectives namely rule metrics for optimization, the problem is presenting the users with solutions with differing trade-offs by incorporating intelligent agents into a data mining system in a cultural algorithm framework and using this agent-mining system for gaining better insight into the social and individual knowledge evolution process. And in turn for evaluating and validating the system and its individuals using the knowledge sources and the rules produced.

\subsection{Aims of the research}

Challenges faced by data mining ${ }^{[3]}$ include enterprise data mining infrastructure, involving domain and human intelligence, supporting parallel and distributed mining, data fusion and preparation, adaptive learning, and interactive mining. The proposed study incorporates some of these challenges including involvement of domain and human intelligence, parallel mining, data fusion, and interactive mining. The proposed study has the following aims:

i. To take data mining (DM) in general and rule induction in particular to the next level by incorporating the strengths of Evolutionary computing (EC) and Agent technology into DM\&KD.

ii. Incorporating Knowledge (memory) into evolutionary systems and enhancing Evolutionary Multi-Objective Optimization (EMOO) with Meta heuristics.

iii. Smooth integration of Agent and DM for interactive data mining so as to convert the social knowledge discovered into actionable social knowledge.

iv. In the process of the interaction of ABSS, EC and DM study the micro and macro trends in social structures through agent evolution and knowledge synthesis by analyzing the resultant evolutionary knowledge stored as snapshots in the different knowledge sources.

The above discussed aims are conceived and a cultural algorithm framework is used in modeling an agent based virtual organization for multi-objective optimization of classification rules. The following section gives a brief overview of EMOO systems for rule mining and a short review of cultural algorithms.

\subsection{Related work}

\subsubsection{EMOO systems for rule mining}

Classification rule mining is the most researched area in data mining since the knowledge produced by classification algorithms is more comprehensible to users. Metrics or properties that are used to evaluate these rules include support, confidence, coverage, understandability, novelty, precision, recall, sensitivity, specificity and accuracy. The rules produced must satisfy at least a subset of these measures if not all to be used as a good classifier. Taking the different measures used for evaluating a rule, the rule mining problem can be thought of as a Multi-Objective Optimization problem. 
Evolutionary algorithms are robust and global search methods that adapt to the environment and can discover interesting knowledge that will be missed by greedy algorithms ${ }^{[4]}$. Also they allow the user to interactively select interesting properties to be incorporated into the objective function providing the user with a variety of choices ${ }^{[5]}$. The use of an EMO algorithm was proposed to search for Pareto-optimal partial classification initially by Iglesia et al., ${ }^{[5]}$ followed by Ishibuchi and Namba ${ }^{[6]}$. Iglesia et al. ${ }^{[5,7]}$, propose the use of EMOO algorithms to allow the user to interactively select a number of interest measures and deliver the best nuggets. A multi-objective genetic algorithm (MOGA) is applied to the problem of partial classification by Reynolds and Iglesia ${ }^{[8]}$. The authors have demonstrated the use of modified dominance relations and clustering techniques to increase the diversity of rules and in the presentation of the potentially large sets of rules generated to the user. An agent-based evolutionary approach is proposed to extract interpretable rule-based knowledge by Wang et al. ${ }^{[9]}$. Dehuri and Mall ${ }^{[10]}$ present a MOGA for mining highly predictive and comprehensible classification rules from large databases. They have proposed a EMOO algorithm called Improved Niched Pareto genetic algorithm (INPGA) for this purpose. Berlanga et al. ${ }^{[1]}$ and Del Jesus et al. ${ }^{[12]}$ present a MOGA for obtaining fuzzy rules for subgroup discovery. Reynolds and Iglesia ${ }^{[13]}$ use multi-objective genetic programming to induce rules. Giusti et al. ${ }^{[14]}$ report a research work that combines evolutionary algorithms and ranking composition methods for multi-objective optimization. In this work candidate solutions are constructed, evaluated and ranked according to their performance in each individual objective. Then rankings are composed into a single ranking which reflects the candidate solutions' ability to solve the multi-objective problem considering all objectives simultaneously. Further Reynolds and De la Iglesia ${ }^{[15]}$ discuss how to adapt a MOO algorithm for the task of partial classification based on a meta-heuristic known as greedy randomized adaptive search procedure (GRASP).

While some EMO algorithms return individual rules using a Michigan style approach there are EMO algorithms that return classification systems or rule sets mostly using Pittsburg style approach. The EMO algorithm by Ishibuchi and Namba ${ }^{[6]}$ is one among many systems that return rule sets. Ishibuchi and Nojima ${ }^{[16]}$ compare fuzzy rules with interval rules using an EMO rule selection method. Further Ishibuchi et al. ${ }^{[17]}$ as an extension of their previous work explain the effectiveness of EMO rule selection as a post processing process. Ishibuchi ${ }^{[18]}$ proposes an EMOA to the design of accurate and interpretable fuzzy rule-based systems and have applied multi-objective genetic fuzzy rule selection. A detailed discussion of evolutionary algorithms for Multi-Objective optimization of classification rules is found in ${ }^{[19]}$.

\subsubsection{EMOO systems that use agents}

$\mathrm{Cao}^{[3]}$ state that the emergence of intelligence in agent-mining interaction may massively strengthen the problem-solving capability of an intelligent system. In the EMOO systems so far discussed only three systems use intelligent agents. The EMOO systems by Wang et al. ${ }^{[9]}$ and Tsang et al. ${ }^{[20,21]}$ use two types of agents namely the Arbitrator agent and the Fuzzy set agent. The Fuzzy set agents initializes its own genes randomly. They are different with one another and selected randomly with the same probability for reproduction. They also autonomously determine their own fuzzy sets information and can consider the interpretability of fuzzy systems. The agents in the system cooperate with each other to exchange information and generate offspring agents. The parent agents and their offspring compete with each other through the arbitrator agent based on the objectives namely accuracy and interpretability and only elite agents move into the next population. Fuzzy set agents obtain information from the arbitrator agent. Arbitrator agent uses the NSGA-II algorithm to evaluate the fuzzy set agents. If Crossover and mutation operations introduce the same rules, the fuzzy set agent will check the offspring fuzzy rule base to delete the redundant rules. Incorporation of agent with DM is said to have improved the performance of the rule mining system.

\subsubsection{A short survey of cultural algorithm}

Cultural algorithm is an evolutionary algorithm which is mostly applied for optimization problems and which has a set of five Knowledge sources for representing various primitive knowledge's and works on the strategy of survival of the fittest. The agents in the system affect the various Knowledge sources (KS's) and the KS's in turn influence the agents thus directing them towards an optimal solution.CA allows agents to interact in many different ways using various forms of symbolic information reflective of complex cultural systems via a shared belief space ${ }^{[22]}$. Cultural algorithm has been used 
for rule induction as well as rule pruning. Sternberg and Reynolds ${ }^{[23]}$ use an evolutionary learning approach based on cultural algorithms to learn about the behavior of a commercial rule-based system for fraud detection. The learned knowledge in the belief space of the cultural algorithm is then used to re-engineer the fraud detection system. Reynolds and Saleem ${ }^{[24]}$ show that the cultural algorithm is more effective than an evolutionary algorithm with only one single-level evolution when they are applied to the problem of finding the new optima in dynamic environments. Lazar and Reynolds have used genetic algorithms and rough sets for knowledge discovery ${ }^{[25]}$. Their work combines decision trees and genetic programming for rule induction. Reynolds and Peng ${ }^{[26]}$ discuss how the learning of knowledge in the belief space ensures the adaptability of cultural algorithms. Reynolds and Saleem ${ }^{[27]}$ further investigate the contributions of different types of knowledge from the belief space in guiding the search toward the best solutions in both deceptive and non-deceptive environments while Reynolds and Ostrowski ${ }^{[28]}$ have used cultural algorithms to evolve strategies for recessionary markets. Reynolds et al ${ }^{[29]}$ investigate the emergence of social patterns in both the population space and the belief space to study how these social patterns reflect role emergence in dynamic environments.

Reynolds et al. ${ }^{[30]}$ use decision trees to characterize location decisions made by early inhabitants at Monte Alban, a prehistoric urban center, and have injected these rules into a socially motivated learning system based on cultural algorithms. They have then inferred an emerging social fabric whose networks provide support for certain theories about urban site formation. Ochoa et al. ${ }^{[31]}$ provide a solution of Logistics Service Based on Data Mining in combination with cultural algorithm, and applied it in optimization of distribution of vehicles within a city. A multi-population multiobjective cultural algorithm adopting knowledge migration is proposed by Guo et al. ${ }^{[32]}$ and has been applied to function-optimization problems. Reynolds and Liu ${ }^{[33]}$ propose an extension of Cultural Algorithms for multi-objective optimization that fully utilizes all of the available categories of knowledge sources and has applied this system to function optimization problem.

Cultural algorithms for multi-objective optimization of rules are rarely found in the literature. Srinivasan and Ramakrishnan ${ }^{[34]}$ have proposed a Cultural Algorithm for Multi-Objective optimization of classification rules. Cao ${ }^{[3]}$ states that the interaction and integration between agent and mining can greatly complement and strengthen each side of both communities. Hence in the current study an attempt has been made to use cultural algorithm for studying the influence of different cognitive traits and in turn the various knowledge sources used by the agents in producing accurate and novel rules. Moreover hybridization of techniques including evolutionary computing, data mining, and agent based social modeling and incorporation of Meta data through the different knowledge sources is enabled by the use of cultural algorithms. CA's have been used to study role emergence in social systems. But in the proposed system the observed roles in those systems and the knowledge sources used by each type of role has been used to define the agents and their traits. Here the agents are distinguished by the type of KS's they use. The agents are then allowed to create knowledge using their traits, the different combinations of KS's. The synthesized knowledge stored in the KS's as a result of evolution is analyzed to gain better insight into the knowledge evolution process both at the micro as well as macro level. This process is termed knowledge reverse engineering. We illustrate the effectiveness of the proposed CA in analyzing evolutionary knowledge using real life applications. The agents in the system take a data source and create classification rules. During this process of knowledge creation, snapshots of the evolutionary knowledge are stored in the different KS's. When termination condition is reached, the knowledge in these KS's are analyzed to observe the type of knowledge used in creating rules with different properties, namely the rule metrics. The contribution of each type of agent in creating rules with different properties is studied. This enables gaining insight into the micro evolutionary knowledge evolution process. The classification rules returned are used to classify unknown test data instances. This is used to evaluate the macro evolution of the social system as a whole. Research gap exists in studying the micro and macro evolutionary knowledge creation process. Also research gap exists in defining a concrete method for evaluating agent based social systems. Knowledge and Innovation forms the basic and necessary asset of any firm, product or service. The proposed Agent Based Virtual Organization for multi-objective optimization of classification rules provides a systematic, principled and concrete method for evaluating ABSS's and for studying the dynamics of a social system. The following section is used to explain the proposed ABVO, its various components and working. 


\section{Agent based virtual organization using cultural algorithm for multi objective optimization of classification rules}

Cultural algorithms are evolutionary systems inspired by social evolution occurring in nature ${ }^{[35]}$. The CA has three components namely the belief space comprising the five knowledge sources, the population space and the communication protocol used to exchange knowledge between the above said spaces comprising of the acceptance and the influence phase. The following section is used to describe the components of the proposed CA.

\subsection{Belief space}

The belief space stores five basic types of information that can be shared cognitively or symbolically ${ }^{[29]}$. The knowledge sources include normative knowledge, situational knowledge, domain knowledge, history knowledge, and topographic knowledge. Reynolds et al. ${ }^{[29]}$ state that this set of knowledge is complete for a given domain in the sense that all available knowledge can be expressed in terms of a combination of these knowledge sources. Moreover the five knowledge sources are said to be used in most animal species in nature and also supported by human social systems. The different knowledge sources are used to store different knowledge's synthesized during evolution depending upon the type of application. In the current implementation of the CA the different knowledge sources are used to store evolutionary knowledge's used in solving rule mining as a multi-objective optimization problem. Moreover an additional KS is added to store individuals which are produced by agents in order to render memory of previous generations to the otherwise memory less evolutionary algorithms. The belief space, the communication protocol including the acceptance and influence strategies and the various features of the evolutionary strategy are discussed in the following sections.

\subsubsection{Normative KS}

Normative Knowledge Source (NKS) contains the ranges of acceptable behaviors. In the current implementation the attributes in the data source and the possible values that the attributes can take are stored in NKS. This information is gathered from the training data set. The normative knowledge source is used to store the maximum and minimum values for numeric attributes. For each nominal or discrete attribute, a separate list is maintained that stores the possible values that the attributes can take. The normative KS is updated during train data set creation and used by the agents during mutation. Once initialized, NKS remains unchanged during evolution.

\subsubsection{Situational KS}

Situational knowledge source (SKS) consists of the best exemplar found along the evolutionary process. It represents a leader for the other individuals to follow. This KS can be updated by storing the best examples at the end of each generation. Also the user can specify a rule schema which can be used by agents for the search of similar/dissimilar individuals to interest the user. In the current study the SKS stores the schema specified by the user as a vector of attribute values.

\subsubsection{Domain KS}

In the basic CA, Domain knowledge records knowledge of domain objects, their relationships, and interactions. In the current version of CA Domain knowledge source (DKS) stores the vector of rule metric values (objective vector) for each rule along with a Rule Identifier (RuleId). Individuals produced by agents are evaluated at the end of each generation and the fitness vector calculated. DKS is updated with these fitness vectors. The fitness vectors in DKS are compared with each other using Pareto optimization strategy to choose elite individuals at the end of each generation. The elite individuals thus chosen are stored in the historical KS.

\subsubsection{Topographical KS}

Topographical knowledge enables recording spatial patterns of behavior. Diversity maintenance strategy is a characteristic of EMOO systems for keeping the solutions uniformly distributed in the Pareto optimal set. Restricted mating, where mating is permitted only when the distance between two parents is large enough, is one technique for maintaining diversity 
of rules ${ }^{[10]}$. In the proposed system Topographic knowledge source (TKS) is used to store the difference or distance between two rules for the purpose of maintaining diversity of rules. This KS is updated at the end of each generation. The topographical knowledge contains a pair of RuleId's and their dissimilarity measure. Since the attributes are discrete in the current implementation, the attribute values in the corresponding positions in the individuals are compared and a value of 0 is assigned to the attributes with similar values and a value of 1 is assigned to dissimilar values. The number of 1's are counted and assigned as the dissimilarity measure for the pair of rules. Only rules in HKS are used to update TKS. Hence topographical KS can be used to create novel and interesting rules by choosing pairs of individuals with maximum dissimilarity measure.

\subsubsection{History KS}

History knowledge source (HKS) records temporal patterns of behavior. In the current study HKS stores in a list, the best individuals (dominators) along with their RuleId's, and are updated at the end of each generation. Evolutionary algorithms are termed as memory less since they do not retain memory of previous generations. However attempts have been made to retain elite individuals of each generation as a separate elite population to render memory to the evolutionary algorithms. Cultural algorithm renders memory to the evolutionary strategy in a systematic way by using the different knowledge sources. History knowledge is updated at the end of each generation. The dominators obtained as a result of comparison of the objective vectors in the DKS are stored in the HKS. HKS holds the elite individuals of each generation thus maintaining memory across generations.

\subsubsection{The Rule KS}

The proposed cultural algorithm is extended to contain the individuals produced during evolution using the Rule KS (RKS). The representation of the individuals in RKS is similar to that of the HKS. Each entry holds a RuleId and the attribute values as a vector. The RuleId is used as a pointer by the other KS's. RKS is added to CA in order to render memory by maintaining good individuals evolved across generations. New rules are added to RKS at the end of each generation. Worst performing individuals can be removed from the system. But sometimes worst parents can produce best off springs. Thus in the proposed system the objective vectors in the DKS for the worst performing individuals are removed, but the individuals themselves are not removed from RKS. This ensures that individuals created during initial generations remain in the population throughout the evolutionary process. Moreover, removal of objective vectors of worst individuals from DKS ensures that worst performers are not involved in the Pareto optimization thus reducing comparison overheads.

\subsubsection{Social agents}

Role emergence has been studied using cultural algorithms found in the literature. The emergent roles from cultural algorithms found in the literature have been used to define cognitive traits of the agents in the proposed ABVO. In the original CA the agents are not distinguished but rather considered as having same properties and are used for exploring the solutions. Role emergence has been studied using the knowledge sources they use. But in the proposed CA for modeling the ABVO, explicitly distinguishes agents with three traits namely imitator, cautious and risk taker defined using the different KS's they use. The agents use these traits in the selection of parents for reproduction. Imitators use SKS and RKS while cautious agents use HKS for choosing parents for mating. Risk takers are explorers and use any of the different KS's at random. A random integer in the range 0 to size of the corresponding KS is generated and the individual in that particular location in RKS or SKS or HKS or TKS are chosen and undergo crossover or mutation. If the KS chosen is TKS then the individuals with the maximum dissimilarity measure is chosen from TKS and reproduction operators are applied to the individuals. This enables creation of diverse set of individuals. Cautious agents use only the historical knowledge source while the imitators use the situational knowledge source and rule knowledge source to create individuals using the example specified by the user. The social agents in the proposed ABVO are allowed to induce rules. The acceptance phase and influence phase allow knowledge exchange between individuals and the organization through the updating and dissemination of knowledge through the belief space. Once the termination condition is reached a process known as reverse engineering is used to analyze the knowledge evolution by analyzing the various KS's which consist of snapshots 
of the evolution. This gives insight into the type of cognitive traits and knowledge sources that are involved in creating knowledge with specific properties. Since innovation creation and knowledge evolution are two processes that are extensively studied in real world firms, in the proposed system novelty measures and accuracy measures are considered as objectives to be optimized.

\subsection{The protocol}

\subsubsection{I nfluence phase}

The influence function decides which knowledge sources influence individuals. Roulette wheel selection based on performance of knowledge sources in the previous generations have been used in the original CA. In the proposed system, selection is left to the agents. In the proposed ABVO the agents use their social trait namely risk taker or imitator or cautious to choose parents for reproduction. Risk takers use knowledge from any of the four knowledge sources namely RKS, HKS, SKS or TKS at random while cautious agents use only the HKS. The imitators choose one parent from SKS and the second parent from RKS to create individuals using the example specified by the user. NKS which stores the possible attribute values is used by all the agents during the mutation operation. The topographical knowledge source enables creation of a diverse set of rules. DKS stores the values of the user specified metrics of the individuals as a fitness vector and thus is used for multi-objective optimization using Pareto comparison. The Rule KS is used to store the individuals (both dominators as well as non-dominators to avoiding losing of good individuals of initial generations) created during evolution. Thus the KS's guide the agents in the evolution process. More social traits can be added to the agents for studying the effect of various traits and knowledge sources on the outcome of the system. The proposed system is thus a complete social system which can be used to model any real time system like a virtual organization or a culture or social communities for studying their micro and macro dynamics and for systematically evaluating such systems.

\subsubsection{Acceptance phase}

The acceptance function determines which individuals and their behaviors can impact the belief space knowledge. Based on selected parameters such as performance, for example, a percentage of the best performers (e.g., top 10\%), can be accepted ${ }^{[22]}$. But since the problem is one of classification rule mining, a threshold value for the rule metrics specified by the user can be used to accept individuals for next generation. Since the current implementation is one of multi-objective optimization, the algorithm produces a set of solutions and the dominators are chosen using Pareto optimization strategy. The process of agent's selection, reproduction, evaluation and updating of belief space forms a generation. At the end of a generation (iteration), the agents return the individuals created by them along with a fitness vector of rule metric values. The knowledge sources are updated with this new knowledge at the end of each generation and thus individuals are accepted into the belief space. The new values in these KS's then influence the population space. Thus the macro evolution takes place.

\subsection{Population space}

\subsubsection{Evolutionary strategy}

Genetic algorithm (GA) is by far the most used evolutionary strategy which is also used in the current study. The population space of the CA is created by the agents using GA. GA is used as the evolutionary strategy to create the population (Solution space comprising of the rules) created using the genetic operators of selection, crossover and mutation. The rules are created by the agents using the genetic operators of selection of individuals from the different knowledge sources using their social traits as explained above. Then the selected individuals undergo crossover and/or mutation depending upon the crossover and mutation rates which are probabilities of crossover and mutation. This rule creation constitutes the micro evolution. Acceptance of the created rules into the belief space using the Pareto optimality optimization strategy constitutes the macro evolution. The virtual organization is evaluated by the rules created by using the accuracy of classification on unknown test data instances and the agents are evaluated based on their contribution in 
producing rules with high metric values. Higher the accuracy in classifying unknown data instances, better the performance of the system. The various attributes of the GA used are discussed below.

\subsubsection{Chromosome representation}

The chosen data records are converted into fixed size chromosomes and represented as a vector of attribute values. The system uses high level encoding where the attribute values are used as they appear in the data source. This reduces the cost of encoding and decoding individuals for creating rules for large data sets. The relational operators are not included in the genotype as found in most algorithms in the literature. Therefore they are not involved in the reproduction which further minimizes the length of the chromosome and thus the time taken for encoding and/or decoding. This representation also avoids use of different types of reproduction operators for different parts of the chromosome.

In the current study the class attribute is also included in the chromosome during the training phase which is also rarely found in rule mining literature. During the test phase classes are assigned to individuals as follows: If more than $75 \%$ of the values in the antecedent part are equal in the rule created and the test data instance then that class is assigned. If more than one rule covers the test instance then the maximum occurring class label that covers the rule is assigned otherwise maximum occurring class in the data set is assigned.

\subsubsection{Population initialization}

Evolutionary systems work on a population of individuals. Population initialization is an important aspect that decides the overall performance of the algorithm. The initial population is created using various procedures. One procedure is seeding where data instances chosen from training data randomly ${ }^{[10]}$ or with the help of the users, are used as initial seeds to fill the population space. Casillas et al. ${ }^{[36]}$ state that the initialization procedure has to guarantee that the initial individuals cover all the input examples from the training data set. In the current study maximum and minimum chromosomes are used as seeds to create initial population. That is, the initialization procedure uses two initial chromosomes as seeds where one chromosome contains the minimum value of all the attributes and the other seed contains the maximum attribute values. These maximum and minimum seeds undergo reproduction and fill the initial population space. However individuals that are not consistent with the data set may be produced. In order to avoid this, the initial population is pruned and individuals that are not consistent with the training data set are removed.

\subsubsection{Reproduction operators}

The operators used for reproduction are selection, crossover and mutation.

Selection strategy: Unlike algorithms found in the EMOO literature, in the proposed system, agents use their social traits in choosing the individuals for reproduction. The agent with the social trait of risk taking chooses rules using any of the knowledge sources at random. The cautious agents choose individuals from historical KS consisting of the elite ones, while imitators use rule schema specified by the user from the situational KS. In this way, knowledge based selection is used rather than random selection. This kind of selection strategy aids in creating not only interesting knowledge but also a diverse set of solutions using the various KS's.

Crossover: Multi point uniform crossover is used. Initially two individuals are chosen at random from the population. A random number which is less than the size of the chromosome is chosen and is taken as the number of crossover points say "c". Then "c" random numbers are generated (less than the chromosome size) and the values at these "c" points are swapped to create two parents.

Mutation: Mutation operates on individual values of attributes in the chromosome. A mutation point is chosen similar to that of the crossover point which is a random integer whose value is less than the chromosome size. The value of the attribute at that point is replaced by another value depending upon the type of the value. For nominal and/or discrete attributes the value to be replaced is chosen at random from a list of available values from NKS. If the value is continuous, a random value in a specified range of minimum and maximum values so far encountered is generated and used for 
reproduction. A list of values for discrete and nominal attributes and lower and upper bound for real valued attributes is stored in NKS.

\subsubsection{Parameters}

The parameters that are to be considered and greatly influence the algorithm performance are the crossover rate, the mutation rate, the population size and the number of generations or the termination condition.

Crossover rate: There are a variety of issues that have been discussed in the literature regarding the crossover rate. Experiments have been carried out using varying crossover rates ranging from $0 \%$ to $100 \%$ but hardly any optimum value has been reported.

Mutation rate: Mutation rate is the rate at which mutation occurs in a generation. A low mutation rate of $1 \%$ and a high mutation rate of $20 \%$ can be found in the literature. However there are discussions about varying the mutation rate as the algorithm proceeds.

Population size: Population sizes ranging from a few dozens to hundreds have been reported. Population size can be varied depending upon the size of the data source.

Stopping criteria: Stopping criteria can be set to a certain number of generations, or it can be set by the user, where the user can stop the algorithm at a point where a satisfactory set of rules have been obtained. Another condition which can be used as stopping criteria is coverage of all the records in the train data set. The algorithm stops when all the records in the train data set have been covered by at least a single rule known as the sequential covering approach. In the current study number of generations specified by the user is used as the stopping criteria. Table 1 summarizes the parameter settings used in the experiments. The parameters used have been chosen as a result of sensitivity analysis performed earlier through various experiments.

Table 1. Parameters

\begin{tabular}{ll}
\hline Parameters & Values \\
\hline Crossover rate & $80 \%$ \\
Mutation rate & $20 \%$ \\
Population size & 500 \\
No. of generations & 25 \\
Stopping criteria & No. of generations \\
Initialization process & Minimum-Maximum seeds \\
Optimization strategy & Pareto optimality \\
Optimization Metrics & Interest, Surprise, Rule difference, Support and Confidence \\
No. of agents & 2 agents of each type \\
\hline
\end{tabular}

Note. Table 1 summarizes the parameter settings used in the experiments. The parameters were chosen as a result of sensitivity analysis experiments carried out earlier

\subsubsection{Optimization strategy/ fitness evaluation}

The optimization or multi-objective optimization strategy forms the acceptance phase of the cultural algorithm. The ultimate objective of Multi-Objective algorithms is to guide the user's decision making, through the provision of a set of solutions that have differing trade-offs between the various objectives ${ }^{[13]}$, and thus the user must be involved in the process of discovering rules. Therefore in the proposed system the user is allowed to control the system by specifying most of the attributes of the system including the rule metrics (objectives), the rule schema, and other parameters as discussed earlier. The user can choose any combination of metrics including coverage, support, confidence, interest, surprise, precision, recall/sensitivity, specificity and a difference measure that stores the difference between the rule and the user specified schema. Coverage and Confidence have been used in the current study. Pareto optimality and ranking 
composition methods are the frequently used optimization strategies. In the current study Pareto optimality has been used as the optimization strategy to select elite individuals.

Pareto optimality is an optimization strategy that uses comparison of the metrics represented as an objective or fitness vector. An individual " $A$ " is said to be better than another individual " $B$ " if " $A$ " is better than " $B$ " in all the metric values or equal to " $\mathrm{B}$ " in all but one metric and better at least in one metric value. This is enabled by the use of Domain KS which stores the fitness vectors of the rules produced. The entries in the DKS are compared with each other and the best performers in all the metrics are returned as dominators. The dominators form the Pareto front found in the Historical KS at the end of the algorithm execution.

\section{Experiments, results and discussion}

\subsection{Methodology}

The proposed Cultural algorithm works as follows:

1) The data set specified by the user is loaded and training $\left(2 / 3^{\text {rd }}\right.$ of data set $)$ and test data $\left(1 / 3^{\text {rd }}\right.$ of data set $)$ sets are created.

2) The rule metrics, the rule schema and the parameters like population size, cross over rate, mutation rate, number of generations and the number of agents of each type are obtained from the user.

3) The data set is converted into chromosome using high level representation.

4) The NKS is updated to hold the list of values of nominal and discrete attributes and minimum and maximum values of numerical attributes.

5) The population is initialized using individuals where one seed contains the minimum value of all the attributes and the other the maximum value of the attributes. These maximum and minimum attribute chromosomes undergo cross over and mutation to create the initial population.

6) The initial population is evaluated to find the values for the user specified metrics and RKS, DKS and TKS are updated as defined in section 3.

7) The best rules which are dominators are chosen to be added to the HKS using the Pareto optimality strategy.

8) The agents use the knowledge in the KS's to create new generations of individuals. Agents use their social traits of cautious (HKS), imitator (SKS and RKS) or risk taker (RKS, SKS, HKS, TKS) to choose rules from different KS's for reproduction.

9) The individuals produced by the agents are added to RKS and evaluated on the user specified metrics. The process of selection, reproduction forms the micro evolution.

10) The belief space is updated by adding the new objective vectors to DKS and the rule difference to TKS. The objective vectors in the DKS are compared and HKS is updated with the dominators.

11) The rule evaluation, acceptance of dominators into the belief space and updating of belief space form the macro evolution. The micro and macro evolution constitute a generation. 
12) When termination condition reaches the individuals in HKS are dominators in the user specified metric values. The algorithm evaluates the rules on test data, presents the rules and rule metrics and stops.

\subsection{Experiments}

Two sets of experiments were conducted to observe the agents in producing accurate rules and novel rules. Support and confidence were considered for accuracy while the novelty measures taken for optimization include interest and surprise measure ${ }^{[37]}$ and rule difference with respect to the rule schema specified by the user. The rule schema difference is introduced as a new measure of novelty in the current study. The rule which has the maximum difference as compared to that of the user specified schema has been used to surprise the user. The metrics are defined as follows:

If $\mathrm{R}: \mathrm{A} \rightarrow \mathrm{C}$ represents the rule. Let $\mathrm{A}$ be the set of all data instances that satisfy the antecedent part and $\mathrm{C}$ be the set of all data instances that satisfy the consequent part. Let $|\mathrm{S}|$ be the cardinality of a set $\mathrm{S}$ and $\mathrm{N}$ the sample size. Then the interest, surprise ${ }^{[37]}$ support and confidence of rule R are defined as in equations (1), (2), (3) and (4) as follows:

$\begin{array}{lll}\text { Interest }(\mathrm{R}) & = & \mathrm{N} * \mid \mathrm{A} \text { and } \mathrm{C}|/| \mathrm{A}|*| \mathrm{C} \mid \\ \operatorname{Surprise}(\mathrm{R}) & = & (\mid \mathrm{A} \text { and } \mathrm{C}|-| \mathrm{A} \text { and }(\operatorname{not} \mathrm{C}))|/| \operatorname{not} \mathrm{C} \mid \\ \text { Support(R) } & = & \mid \mathrm{A} \text { and } \mathrm{C} \mid / \mathrm{N} \\ \text { Confidence(R) } & = & \mid \mathrm{A} \text { and } \mathrm{C}|/| \mathrm{A} \mid\end{array}$

The Schema-Rule difference is calculated by comparing the corresponding attribute values in the user specified schema and the rule and assigning a 1 if the values are different and 0 if not. This is possible since the attribute values are discretized. The difference is then calculated by summing the 1's. The accuracy of a rule on the test data set is defined by the ratio of number of data instances correctly classified by $\mathrm{R}$ to the total number of instances in the data set. The Interest and Surprise metrics used in the proposed system are defined ${ }^{[37]}$. Interest measures the dependency and gives privilege to rare patterns and takes values in the interval $(0, \infty)$. Surprise is a measure which enables search of surprising data instances and takes values in the interval $[0, \infty)$.

The two experiments were run ten times each with different train and test data sets for cross validation. The Wisconsin breast cancer data set from the UCI machine learning repository ${ }^{[38]}$ has been used in the study. The Wisconsin Breast Cancer Data set consists of 699 instances with two classes. Each instance consists of 9 continuous attributes. The attributes are assigned integer values between 1 and 10 . The data set contains 16 instances with missing values which have been removed and remaining 683 cases are used.

\subsection{Results and discussion}

Table 2 summarizes the performance of the system in classifying unknown test data instances taking the two sets of objectives. It is interesting to observe from Table 2 that the rules returned by the system using novelty measures of interest, surprise and rule difference perform as good as accurate rules in classification accuracy. This means that novel rules are also accurate. However when both the accuracy and novelty measures are taken together, the number of dominators returned by the algorithm is less with an average of 11.5 rules as observed from HKS. This observation suggests that not all accurate rules are novel. Another interesting suggestion is that novelty measures can be incorporated with accuracy metrics to produce a compact set of rules to increase the comprehensibility of the rule set returned (less number of rules means more comprehensible). Further incorporation of novelty measures also reduces the time taken by the algorithm to complete. Table 3 summarizes the average contributions in percentage over ten runs of the algorithm of the three different types of agents in producing novel rules taking Interest, Surprise, Rule difference as objectives for optimization. 
Table 2. Performance of the algorithm (Average over ten runs)

\begin{tabular}{lcccc}
\hline Rule Metrics & RKS & HKS & $\begin{array}{l}\text { Time } \\
\text { (seconds) }\end{array}$ & $\begin{array}{l}\text { Accuracy\% } \\
\text { (test data) }\end{array}$ \\
\hline $\begin{array}{l}\text { Interest, Surprise, } \\
\text { Rule difference } \\
\begin{array}{l}\text { Support, Confidence, } \\
\text { Interest, Surprise }\end{array}\end{array}$ & 211.5 & 16.2 & 13.15 & 94.87 \\
\hline
\end{tabular}

Note. Table 2 summarizes the performance of the algorithm in classifying unknown test data instances for the two combinations of objectives.

Table 3. Agent contribution in producing novel rules (Average over ten runs)

\begin{tabular}{ll}
\hline Agent type & Contribution \% \\
\hline Cautious & 45.71 \\
Imitator & 30.1 \\
Risk Taker & 24.19 \\
\hline
\end{tabular}

Note. Table 3 summarizes the average contributions of the three different types of agents in producing novel rules taking Interest, Surprise, Rule difference as objectives for optimization.

Table 4. Agent contribution in producing accurate and novel rules (Average over ten runs)

\begin{tabular}{ll}
\hline Agent type & Contribution \% \\
\hline Cautious & 55.5 \\
Imitator & 21.55 \\
Risk Taker & 22.06 \\
\hline
\end{tabular}

Note. Table 4 gives the average contribution in percentage over ten runs of the algorithm for each agent type in producing accurate and novel rules taking Support, Confidence, Interest and Surprise as objectives for optimization.

It is an interesting observation from Table 3 and 4 that cautious agent which use HKS leads in producing both novel rules as well as accurate rules. $45.71 \%$ of the novel rules returned and $55.5 \%$ of the rules taking together both accuracy and novelty as objectives have been created by cautious agents. Cautious agents using the knowledge in HKS contribute to nearly $50 \%$ of the elite population. There is a slight variation however between Imitator and risk taker. It is an interesting phenomena that imitator contributes more to producing novel rules with an average of $30.10 \%$. Whereas it is worthy to note that both imitator and risk taker are more or less equal in producing novel and accurate rules. While Table 3 and 4 shows the average contributions of the agents, Figure 1 and 2 give the spread of performance of each agent type in producing novel rules and accurate rules over ten runs of the algorithm using box plots. Figure 1 and 2 also show the consistency of the agent performance in the various runs. Figure 1 gives the spread of performance of each agent type in producing novel rules over ten runs using box plots. Figure 2 gives the spread of performance of each agent type in producing novel and accurate rules over ten runs using box plots.

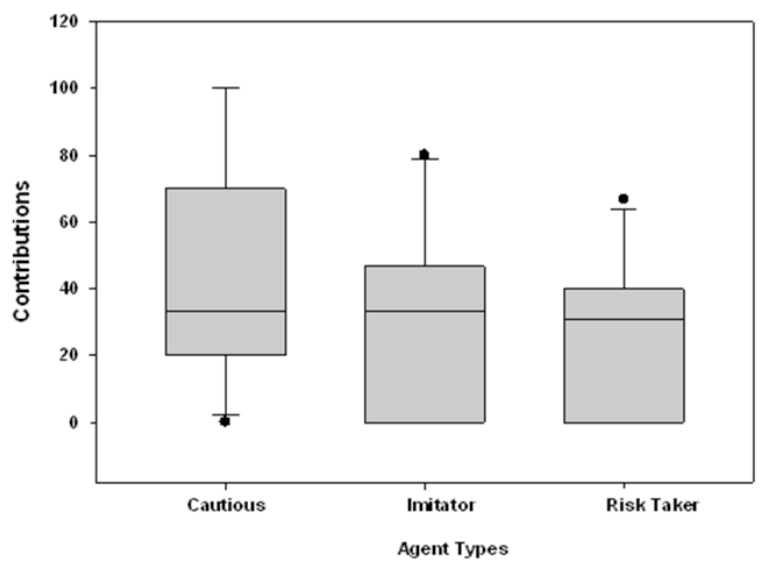

Figure 1. Agent types and contributions towards novel rules 


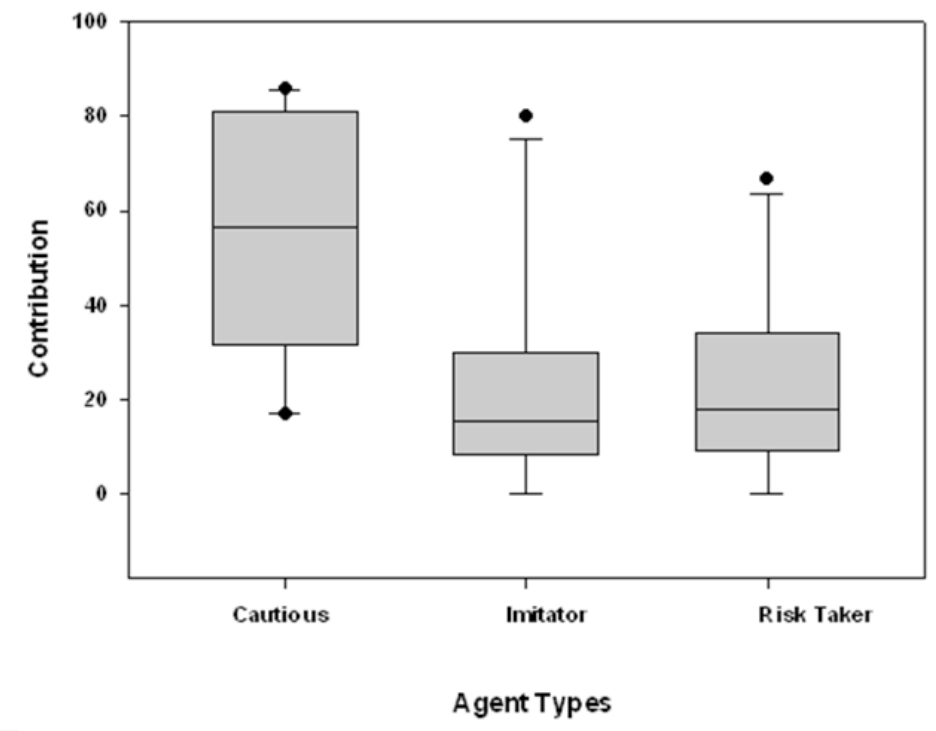

Figure 2. Agent types and contributions towards Accurate and Novel rules

It is interesting to note again from Figure 1 and 2 that agent's contribution in producing novel rules shows very small difference while there is significant difference in the contributions of agents in producing accurate rules. It is also worth noting that in certain runs of the algorithm all the three types of agents have produced as low as zero rules when considering novelty measures. But when considering accurate rules cautious agents perform well with a maximum of $100 \%$ in some runs.

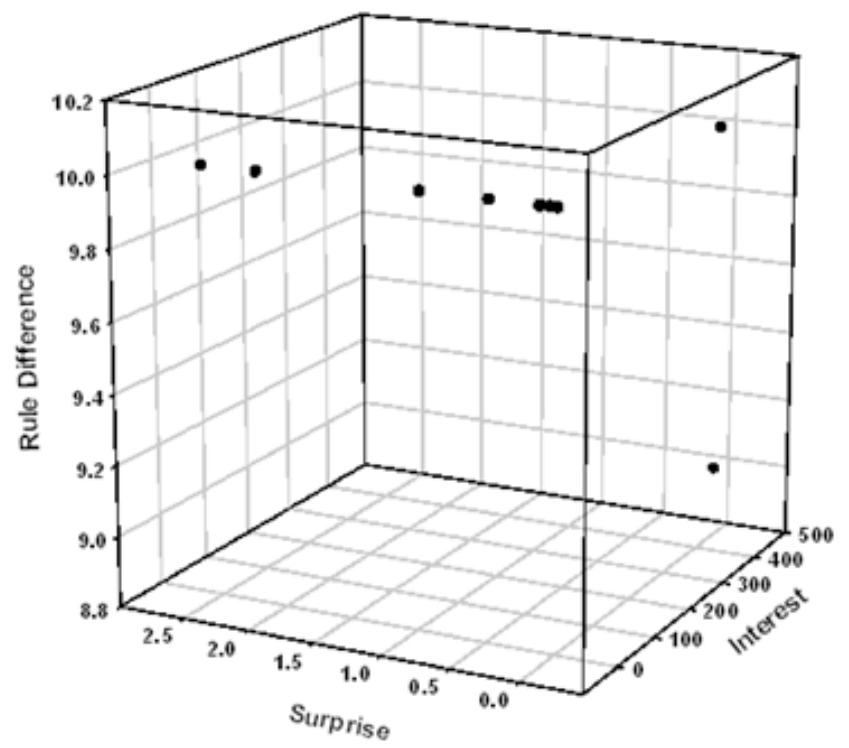

Figure 3. Sample Pareto front in novelty measures 


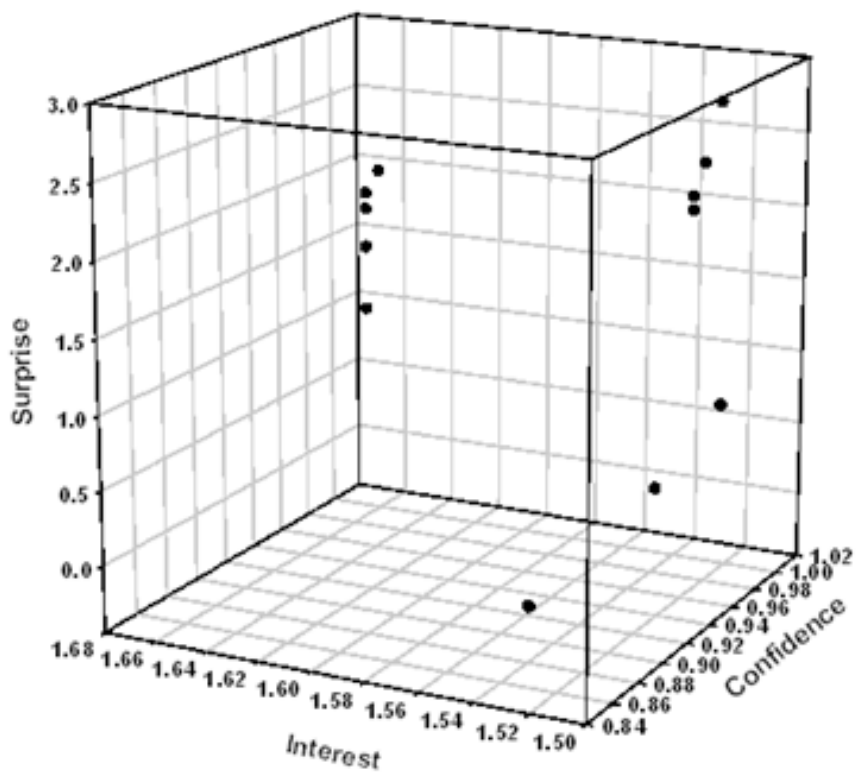

Figure 4. Sample Pareto front in accuracy and novelty measures

Figure 3 shows sample Pareto front in novelty measures of Interest, Surprise and Rule difference while Figure 4 shows a sample Pareto front in Accuracy measure of Confidence and Novelty measures of Interest and Surprise taken together. It can be observed from Figures 3 and 4 that the algorithm is good in producing a good set of rules optimized in all the objectives whether novelty or accuracy metrics are considered. But the Interest and Surprise metrics can take values ranging from 0 to Infinity. It is interesting that when considering only the novelty metrics of Interest, Surprise and Rule difference, the algorithm was able to create rules with high Interest measure with a value of infinity for certain rules and high rule difference measures. The surprise value ranged from 0.1 to infinity. But an interesting observation was that when interest value was maximum (infinity), the surprise value was minimum (0.19) and vice versa. This needs further investigation. However when accuracy and novelty measures were taken together for optimization the algorithm was able to produce accurate rules with confidence ranging from 0.86 to 1.0 and support ranging from 0.15 to 0.48 but the novelty measures, interest ranged from 1.51 to 1.66 , and surprise value ranged from 0.15 to 2.77 in most of the cases. This gives rise to a question, "Are accurate rules that are termed interesting are really not so interesting?" needs further investigation. Also the surprise measure has a maximum value of 2.77 while it can be as high as infinity which needs further analysis and experimentation.

\subsection{Contributions of the proposed system}

The proposed hybrid ABVO contributes to data mining, in particular rule mining as a multi-objective optimization problem, to evolutionary computing and to agent based social modeling.

\subsubsection{Contributions to agent based social modeling}

i. $\quad \mathrm{CaO}^{[3]}$ states that agent-mining fosters a synergy between agent and data mining from different dimensions, for instance, resource, infrastructure, learning, knowledge, interaction, interface, social, application and performance. The proposed extended cultural algorithm enables studying the dynamics of a real world social system both at the micro and macro levels by analyzing the KS's in the belief space.

ii. It provides a systematic and principled approach to evaluate agent based systems with the outcomes namely the rules produced by optimizing the different metrics specified by the user. 


\subsubsection{Contributions to data mining}

i. The proposed system allows the user to specify the rule metrics and a schema that are used by the agents to produce the rules. Hence user knowledge is incorporated into the system.

ii. The system presents the user solutions with differing tradeoffs to choose from.

\subsubsection{Contributions to evolutionary computing}

i. The user is also allowed to mention the various evolutionary parameters namely crossover and mutation rates, population size, and the number of generations for studying the implication of these parameters to a rule mining system.

ii. Knowledge is incorporated through the different KS's to evolutionary algorithms which are otherwise memory less and uses blind search.

\section{Conclusion}

An Agent Based Virtual Organization for studying the knowledge evolution in social systems is proposed. The system is modeled using a cultural algorithm framework. Moreover the hybrid intelligent system is used in solving classification rule mining as a Multi-Objective Optimization problem. This provides a concrete and systematic approach for evaluating the agent based social system by analyzing the knowledge sources that store the evolutionary snap shots of knowledge and the rules created by the system. From the results it can be observed that the system performs well in creating good set of rules optimized in the user specified objectives and in classifying unknown data instances. The Wisconsin breast cancer data set from the UCI machine learning repository ${ }^{[38]}$ has been used for illustrating the proposed system. However more experiments to find the influence of the various parameters, the size and type of the data set, the ratio of agents on the micro and macro evolution and the overall performance of the system are required. Moreover in the current implementation the number of agents of each type is taken to be equal with 2 agents of each type. From the results discussed, the questions that need to be answered are:

1) Will the algorithm converge to better solutions in less time if the number of cautious agents is increased?

2) What is the optimal ratio of agent type that will produce good set of rules optimized in various combinations of the user specified metrics?

3) Will there be difference in the contributions of agents for other data sets?

4) What is the influence of the size and dimension of the data sets and the number of metrics for optimization on agent performance?

These are some of the questions that need further exploration.

\section{Future enhancements}

The agents in the current system are not designed in a manner to change strategies during evolution. This will be the proposed future enhancement. The agents will be incorporated with more functional capabilities so as to analyze the evolved knowledge and change both their beliefs and norms so as to balance benefits and costs, and maximize their return while minimizing the risk. Moreover other cognitive and social traits can be added to make the system more real world like. 


\section{References}

[1] Wang Fei-Yue Daniel Zeng, Kathleen M. Carley, Wenji Mao. Social Computing: From Social Informatics to Social Intelligence. IEEE Intelligent Systems. March/April. 2007; 79-83.

[2] Li Xiaochen, Mao WenjiZeng Daniel, Wang Fei-Yue. Agent-Based Social Simulation \& Modeling in Social Computing. C.C. Yang et al. (Eds.): ISI 2008 Workshops. LNCS 5075.Berlin Heidelberg: Springer-Verlag; 2008: 401-412.

[3] Cao L. Introduction to agent-mining interaction \& integration. In: L. Cao (Ed.) Data mining \&multiagent integration. LLC 2009.Berlin: Springer; 2009: 3-36.

[4] FreitasA A. A review of evolutionary algorithms for data mining.Soft computing for knowledge discovery \& data mining. USA: Springer; 2007: 79-111.

[5] De la Iglesia B, Philpott MS, Bagnall A J,Rayward-Smith V J. Data mining rules using Multi-Objective evolutionary algorithms. In: Proceedings of 2003 IEEE congress on evolutionary computation. 2003; 1552-1559.

[6] Ishibuchi H, Namba S. Evolutionary multiobjective knowledge extraction for high-dimensional pattern classification problems, Parallel Problem Solving from Nature-PPSN VIII, LNCS 3242.Berlin: Springer; 2004: 1123-1132.

[7] De la Iglesia B, Reynolds Alan, Rayward-Smith Vic J. Developments on a Multi-Objective meta-heuristic (MOMH) algorithm for finding interesting sets of classification rules. In: Proceedings of third international conference on Evolutionary Multi-criterion Optimization, EMO2005, LNCS 3410. Berlin: Springer; 2005: 826-840.

[8] Reynolds A P, De la Iglesia B. Rule induction using Multi-Objective meta-heuristic: encouraging rule diversity, In: Proceedings of IJCNN. 2006: 6375-6382.

[9] Wang H, KwongS, Jin Y, Wei W, Man K F. Agent based evolutionary approach for interpretable rule-based knowledge extraction. IEEE Trans Syst Man Cybern. 2005; 35(2): 143-155. http://dx.doi.org/10.1109/TSMCC.2004.841910

[10] Dehuri S, Mall R. Predictive \& comprehensible rule discovery using a Multi-Objective genetic algorithm. Knowl Syst. 2006; 19: 413-421. http://dx.doi.org/10.1016/j.knosys.2006.03.004

[11] Berlanga F, Del Jesus, M J, Gonzalez P, Herrera F, Mesonero M. Multi-Objective evolutionary induction of subgroup discovery fuzzy rules: a case study in marketing. In: P. Perner (Ed.) ICDM 2006, LNAI 4065. Berlin: Springer; 2006; 337-349.

[12] Del Jesus MJ, Gonzalez P, Herrera F. Multi-Objective genetic algorithm for extracting subgroup discovery fuzzy rules. In: Proceedings of the 2007 IEEE symposium on computational intelligence in Multi-Criteria Decision Making (MCDM 2007). 2007: $50-57$.

[13] Reynolds A P, De la Iglesia B. Rule induction for classification using Multi-Objective genetic programming. In: Proceedings of 4th international conference on Evolutionary Multi-criterion Optimization, LNCS 4403. Berlin: Springer; 2007; 516-530.

[14] Giusti R, Gustavo EA, Batista PA, Prati Ronaldo C. Evaluating ranking composition methods for multiobjective optimization of knowledge rules. In: Proceedings of eighth international conference on Hybrid Intelligent Systems. 2008 : $537-542$. http://dx.doi.org/10.1109/HIS.2008.154

[15] Reynolds A P, De la Iglesia B. A Multi-Objective GRASP for partial classification. Soft Comput. 2009 ; $13(3)$ : $227-243$. http://dx.doi.org/10.1007/s00500-008-0320-1

[16] Ishibuchi H, Nojima Y. Comparison between fuzzy \& interval partitions in evolutionary Multi-Objective design of rule-based classification systems. In: Proceedings of the 2005 IEEE international conference on Fuzzy systems. 2005: 430-435.

[17] Ishibuchi H, Kuwajima I, Nojima Y. Multi-objective classification rule mining, Natural computing series. Springer, Berlin. 2007; 219-240.

[18] Ishibuchi H. Evolutionary Multi-Objective design of fuzzy rule-based systems. In: Proceedings of the 2007 IEEE symposium on Foundations of Computational Intelligence (FOCI 2007). 2007: 9-16. http://dx.doi.org/10.1109/FOCI.2007.372141

[19] Srinivasan Sujatha, Ramakrishnan Sivakumar. Evolutionary Multi-Objective optimization for rule mining: a review. Artificial Intelligence Review. 2011; 36(3): 205-248. http://dx.doi.org/10.1007/s10462-011-9212-3

[20] Tsang C-H, Kwong S, Wang H. Anomaly intrusion detection using Multi-Objective genetic fuzzy system \& agent-based evolutionary computation framework. In: Proceedings of the fifth IEEE international conference on data mining (ICDM'05). 2005: 789-792.

[21] Tsang C-H, Kwong S, Wang H. Genetic-fuzzy rule mining approach \& evaluation of feature selection techniques for anomaly intrusion detection. Pattern Recogn. 2007; 40: 2373-2391. http://dx.doi.org/10.1016/j.patcog.2006.12.009

[22] Reynolds R G, Bin Peng,Mostafa Ali. The Role of Culture in the Emergence of Decision-Making Roles, An example using Cultural Algorithms. Complexity, Wiley Periodicals, Inc. 2007; 13(3): 27-42.

[23] Sternberg M, Reynolds R G. Using cultural algorithms to support re-engineering of rule-based expert systems in dynamic environments: A case study in fraud detection. IEEE Trans. Evol. Comput. 1997; 1(4): 225-243.

http://dx.doi.org/10.1109/4235.687883 
[24] Reynolds R G, Saleem S. The impact of environmental dynamics on cultural emergence. In L. Booker et al., (Eds.) Perspectives on Adaptation in Natural \& Artificial Systems-Essays in Honor of John Holland. London, U.K.: Oxford Univ. Press; 2004 : $253-280$.

[25] Lazar A,\& Reynolds R G. Heuristic Knowledge Discovery for Archaeological Data Using Cultural Algorithms \& Rough Sets. Heuristics \& Optimization for Knowledge Discovery.2002; 2.

[26] Reynolds R G, Peng B. Cultural algorithms: Knowledge learning in dynamic environments. In Proc. IEEE Int. Congr. Evol. Comput. 2004: 1751-1758.

[27] Reynolds RG,SaleemS. Function optimization with cultural algorithms in dynamic environments. In Proceedings of IEEE Particle Swarm Optimization Workshop. 2001: 63-79.

[28] Reynolds R G, Ostrowski D. Using cultural algorithms to evolve strategies for recessionary markets. In Proceedings of IEEE Int. Congr.Evol.Comput. 2004: 1780-1785.

[29] Reynolds R G, Bin Peng, Mostafa Ali. The Role of Culture in the Emergence of Decision-Making Roles, An example using Cultural Algorithms. Complexity, Wiley Periodicals, Inc. 2007; 13(3): 27-42.

[30] Reynolds RG, Mostafa Ali, ThaerJayyousi. Mining the Social Fabric of Archaic Urban Centers with Cultural Algorithms, IEEE Computer. 2008: 64-72.

[31] Ochoa Alberto, García Yazmani, Yanez Javier, Teymanoglu Yaddik. Using Cultural Algorithms to Improve Intelligent Logistics. E.S. Corchado Rodriguez et al. (Eds.): HAIS 2010, Part II, LNAI 6077, pp. 127-134, 2010. Berlin Heidelberg: Springer-Verlag; 2010.

[32] Guo Yi Nan, Cao Yuan, Liu Dan Dan. Multi-Population Multi-Objective Cultural Algorithm, In Han et al., (Eds.), Advanced Materials Research. October 2010; (156-157): 52-55.

[33] Reynolds R G, Dapeng Liu. Multi-Objective Cultural Algorithms. IEEE Congress on Evolutionary Computation (CEC2011). 2011; 1233-1241.

[34] Srinivasan Sujatha, Ramakrishnan Sivakumar. Multi-Objective Optimization of classification rules using Cultural Algorithms. Procedia Engineering. Elsevier. 2012; 30 (0): 457-465.

[35] Reynolds RG.An introduction to cultural algorithms. In: Proceedings of the 3rd Annual Conference on Evolutionary Programming; World Scientific: River Edge, NJ; 1994: 131-139.

[36] Casillas J, Orriols-Puig A, Bernad_o-Mansilla E. Toward evolving consistent, complete, and compact fuzzy rule sets for classification problems. In: proceedings of 3rd international workshop on genetic and evolving fuzzy systems, Witten-Bommerholz, Germany. 2008; 89-94.

[37] Khabzaoui M, Dhaenens C, Talbi EG. Combining evolutionary algorithms \& exact approaches for Multi-Objective knowledge discovery. RAIRO Oper Res. 2008; 42: 69-83. http://dx.doi.org/10.1051/ro:2008004

[38] Newman D, Hettich S, Blake C, Merz C. UCI repository of machine learning databases, Department of Information \& Computer Science, University of California at Irvine. 1998. http:/www.ics.\%20uci.edu/?mlearn/MLRepository.html 